\title{
Comparative Evaluation of Gingival Zenith Deviation from the Vertical Bisected Midline and Apicocoronal Displacement of the Zenith of Lateral Incisor from Gingival Zenith Level: A Cross-sectional Study
}

\author{
Shivanand Aspalli ${ }^{1}$, Susan Abraham ${ }^{2}$, Reetika Gaddale ${ }^{3}$, Syeda Shagufta N ${ }^{4}$, Ashrita Agrawal ${ }^{5}$, Minam Tamuk ${ }^{6}$
}

\begin{abstract}
Aim and objective: To measure the deviation of the gingival zenith (GZ) of maxillary anterior teeth with the vertical bisected midline (VBM) and apicocoronal displacement of lateral incisor zenith from $\mathrm{GZ}$ level of central incisor and canine.

Materials and methods: For 50 periodontally healthy subjects (age range 18-50 years) having all anterior teeth, alginate impressions were made and a diagnostic cast was obtained. Gingival zenith was marked and its deviation from the VBM was measured for the maxillary anterior teeth using the method described by Chu and Stappert. The apicocoronal distance of the GZ point of the lateral incisor was measured from the GZ level of the central incisor and canine. The data were tabulated and subjected to statistical analysis.

Results: Gingival zenith deviation was greater in the central incisor, followed by lateral incisor and canine. The mean apicocoronal displacement of the GZ of the right lateral incisor was $0.606 \pm 0.417 \mathrm{~mm}$ and the left lateral incisor was $0.537 \pm 0.414 \mathrm{~mm}$ from the GZ level.

Conclusion: Gingival zenith deviation can be mesial or distal and is tooth-dependent. It was found that in the majority of cases, lateral incisors showed coronal displacement of GZ from the GZ line.
\end{abstract}

Keywords: Gingival zenith, Gingival zenith deviation, Gingival zenith line, Maxillary anterior teeth, Vertical bisected midline. International Journal of Prosthodontics and Restorative Dentistry (2021): 10.5005/jp-journals-10019-1317

\section{INTRODUCTION}

Multidisciplinary integration is necessary to achieve esthetical and functional results in simple and complex dental rehabilitation. Planning and establishing the correct timing of the involved procedures increase treatment predictability. While performing the oral rehabilitation procedures, either we can mimic the contralateral teeth form, alignment, and proportion or design it based on the esthetic principles and characteristics of individual teeth. Designing a harmonious smile would require interventions from individual dental specialties, such as surgical, orthodontic, and restorative procedures. To substantiate the necessity of such interventions, the gingival contour must be evaluated, and establishing the correct gingival zenith (GZ) aids in the treatment planning as well as in the following dental procedures. ${ }^{1}$

Gingival zenith is the most apical portion of the gingival margin. Studies have shown that in maxillary central incisors, it is usually distally displaced and in maxillary lateral incisors and canines, it is usually centralized. ${ }^{1}$

The GZ plays a pivotal role in the case of cosmetic dentistry. The form and contour of gingival tissues along with the overall esthetics form the important factors that define an esthetic smile. The visibility of the dentogingival interface in the anterior region thus pose an esthetic challenge to dental procedures in this region. The physiologic gingival contour has been defined as the one in which the interdental area is conical and coronally positioned to the buccal and lingual (or palatal) plates of bone, which have a parabolic shape and flow smoothly from the interdental area; that follows the shape of the cementoenamel junction (CEJ), allowing a thin, scalloped, knife-edged gingival contour with pyramid- \begin{tabular}{l}
\hline \hline${ }^{1-6}$ Department of Periodontology and Oral Implantology, AME'S \\
Dental College and Hospital, Raichur, Karnataka, India
\end{tabular} Corresponding Author: Susan Abraham, Department of Periodontology and Oral Implantology, AME'S Dental College and Hospital, Raichur, Karnataka, India, Phone: +91 8547033003, e-mail: susanab1992@gmail.com

How to cite this article: Aspalli S, Abraham S, Gaddale R, et al. Comparative Evaluation of Gingival Zenith Deviation from the Vertical Bisected Midline and Apicocoronal Displacement of the Zenith of Lateral Incisor from Gingival Zenith Level: A Cross-sectional Study. Int J Prosthodont Restor Dent 2021;11(2):88-92.

Source of support: Nil

Conflict of interest: None

shaped papillae that fill the interproximal space. This knife-edged, festooned marginal gingival contour is primarily affected by the degree of concavity and convexity of the tooth surface. ${ }^{2}$

It is very important to locate the gingival zenith point (GZP) especially when carrying out esthetically important procedures such as changing mesiodistal dimensions or in cases of diastema closure. Also, while trying to reduce the exaggerated triangular form of teeth or bringing about the illusion of bodily movement by altering the position of the $\mathrm{GZ}$, or for correction of tooth angulation, locating the $\mathrm{GZ}$ is very important. ${ }^{3}$

The contour of gingival margins, along with the smile and facial components must all be in harmony therefore any deviations must be corrected by surgical or orthodontic treatment especially when the patient presents a high lip line.

(c) Jaypee Brothers Medical Publishers. 2021 Open Access This article is distributed under the terms of the Creative Commons Attribution 4.0 International License (https://creativecommons.org/licenses/by-nc/4.0/), which permits unrestricted use, distribution, and non-commercial reproduction in any medium, provided you give appropriate credit to the original author(s) and the source, provide a link to the Creative Commons license, and indicate if changes were made. The Creative Commons Public Domain Dedication waiver (http://creativecommons.org/publicdomain/zero/1.0/) applies to the data made available in this article, unless otherwise stated. 
Thus the study aimed to measure the deviation of GZ of maxillary anterior teeth from the vertical bisected midline (VBM) and also to measure the GZ distance (apicocoronally) of lateral incisor from gingival zenith level (GZL) of central incisor and canine.

\section{Materials and Methods}

Patients presenting to the Department of Periodontics and Oral Implantology, AME's Dental College and Hospital, Raichur, Karnataka, India fulfilling the inclusion and exclusion criteria were enrolled in the study. The study was explained to the patient and written informed consent was taken and an ethical clearance certificate was also obtained from the institution.

The inclusion criteria included periodontally healthy subjects of 18 years and above, having all six maxillary anterior teeth. The exclusion criteria included subjects having crown restoration or prosthesis in maxillary anterior teeth, those with spacing, crowding, or midline diastema, those receiving medications with any known effect on periodontal soft tissue, those undergoing orthodontic treatment or with the previous history of orthodontic treatment, and those with any history of periodontal surgery in the maxillary anterior region. Thus, 50 periodontally healthy subjects ( 45 females, 5 males) were included in the study.

Alginate impressions were made and poured with dental stone and a diagnostic cast was obtained for each subject. On each cast, the following points were marked, i.e., VBM, GZP, and GZL. All the measurements were done using a digital caliper.

The GZP and VBM was marked according to the method given by Chu et al. in 2009. ${ }^{4}$ Vertical bisected midline was marked for each tooth from canine to canine using the following method. The mesiodistal width of the tooth was measured at two reference points. The two reference points taken were the proximal incisal contact area position (ICAP) and the apical contact area position (ACAP) (Fig. 1). Both the widths were divided in half, and the center point for each was marked. The two center points were then further extended toward the gingival contour of the crown to obtain a line and thus the VBM was located (Fig. 2).

The highest point of the gingival contour for each tooth gives the GZP. Thus, the GZP was identified and marked for all six maxillary anterior teeth (Fig. 1). The distance of the GZP to the VBM was measured with a digital caliper for each tooth, including the central incisors, lateral incisors, and canine teeth (Fig. 3).

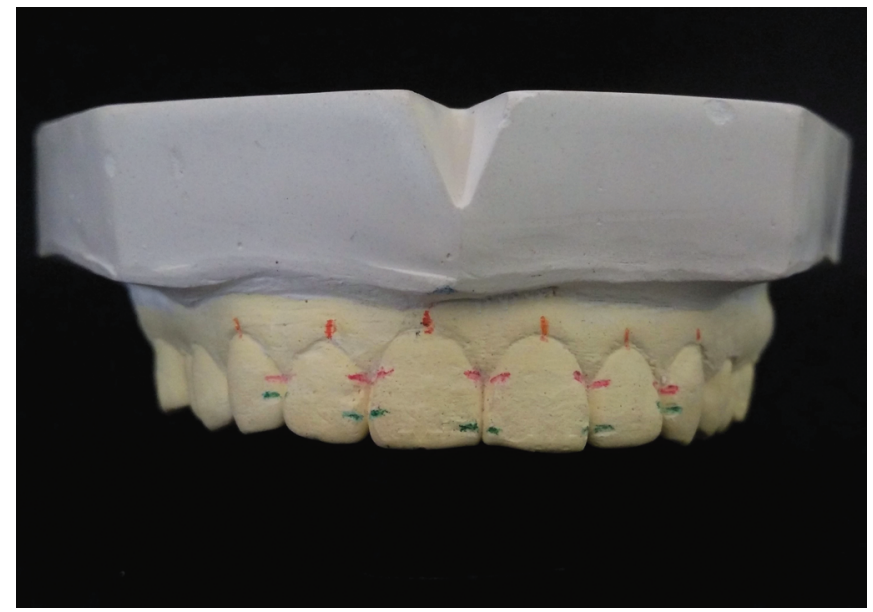

Fig. 1: Marking of gingival zenith and two reference points (proximal incisal contact area position and the apical contact area position)
A gingival line (tangent) was drawn by joining the GZP of the maxillary central to the canines to obtain GZL. The distance of the GZP of the lateral incisor was measured from the GZL in an apicocoronal direction. This was then compared with the GZL of central incisor and canine (Fig. 4).

The data obtained were tabulated and subjected to statistical analysis using the software (SPSS version 20, IBM Corp., Armonk, NY, USA). The data obtained were statistically analyzed using a repeated $T$ test.

\section{Results}

Fifty casts were evaluated for deviation of GZ of all the maxillary anterior teeth from the VBM. Both mesial and distal deviations were seen, although the majority showed distal deviation. The apicocoronal displacement of GZ of lateral incisor from the GZL of central incisors and canine was also evaluated.

Table 1 shows the deviation of GZP from the VBM of central incisors, it was found that in $74 \%$ of right central incisors (37 out of 50 , mesial deviation-12, distal deviation-25) and $68 \%$ of left central incisors (34 out of 50, mesial deviation-4, distal deviation-30), the GZ showed deviation. The mean deviation was $0.307 \pm 0.605 \mathrm{~mm}$ for right central incisors and $0.229 \pm 0.258 \mathrm{~mm}$ for left central incisors. The difference in the mean GZ deviation from VBM between right and left central incisors was not statistically significant ( $p$ value $=$ 0.345).

In the case of lateral incisors, Table 1 shows that in $64 \%$ of right lateral incisors (32 out of 50, mesial deviation-18, distal deviation-24) and $78 \%$ of left lateral incisors (39 out of 50, mesial deviation-3, distal deviation-36), the GZP showed deviation from the VBM. The mean deviation was $0.159 \pm 0.194 \mathrm{~mm}$ for right lateral incisors and $0.276 \pm 0.265 \mathrm{~mm}$ for left lateral incisors. The difference in the mean GZ deviation from VBM between right and left lateral incisors showed a highly statistically significant variation ( $p$ value $=0.005$ )

Table 1 shows the deviation of GZ point from the VBM of canine and it was found that in $66 \%$ of right canines ( 33 out of 50 , mesial deviation-19, distal deviation-14) and $50 \%$ of left canines ( 25 out of 50, mesial deviation-6, distal deviation-19), the GZ showed deviation. The mean deviation was $0.173 \pm 0.234 \mathrm{~mm}$ for right canines and $0.195 \pm 0.269 \mathrm{~mm}$ for left canines. The difference in the mean GZ deviation from VBM between right and left canine was not statistically significant ( $p$ value $=0.625$ ).

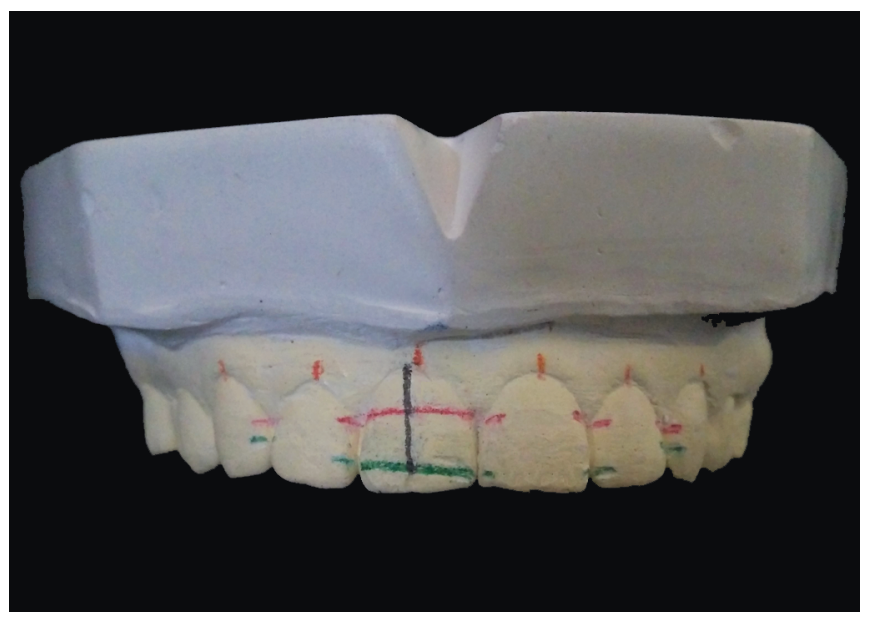

Fig. 2: Location of vertical bisected midline using the reference points 


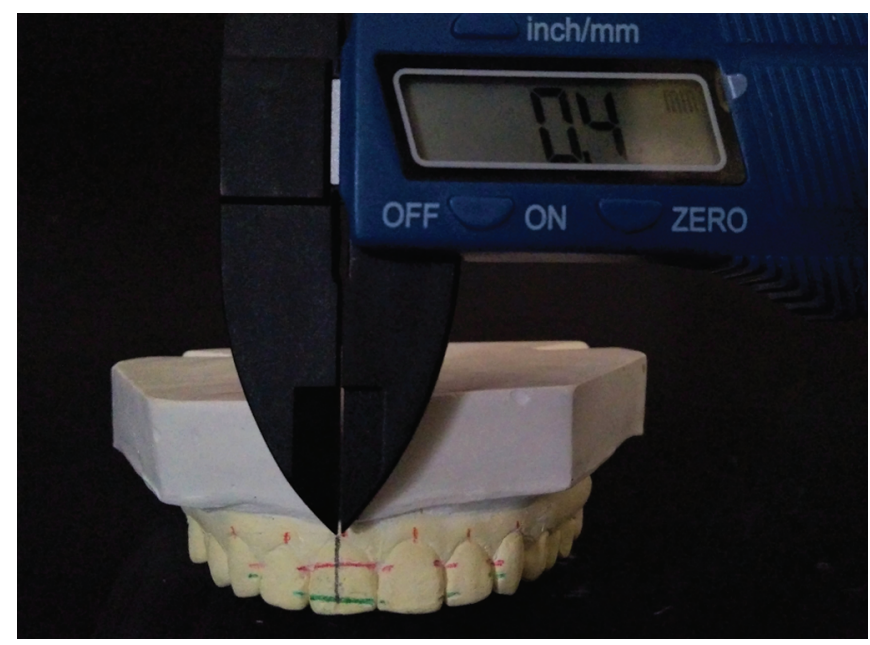

Fig. 3: Measurement of variation of gingival zenith from vertical bisected midline using Vernier caliper

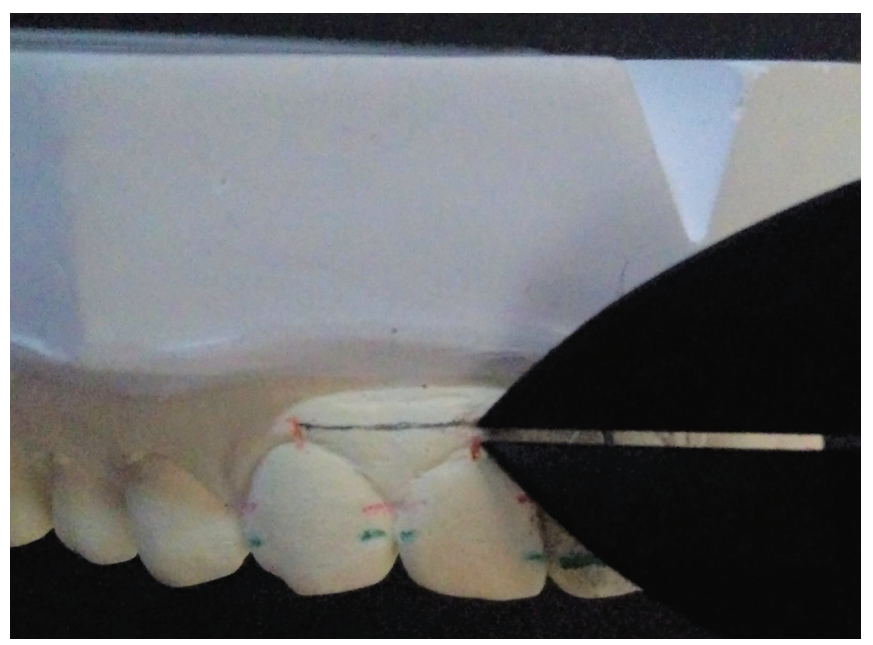

Fig. 4: Measurement of variation of gingival zenith of lateral incisor from gingival zenith level using Vernier caliper

Table 1: Mean deviation of gingival zenith point of the individual tooth from vertical bisected midline

\begin{tabular}{lllllllll}
\hline Tooth no. & $\begin{array}{l}\text { No. of teeth showing } \\
\text { deviation (out of 50) }\end{array}$ & Mean \pm SD & $p$ value & $\begin{array}{l}\text { Standard error } \\
\text { mean }\end{array}$ & $\begin{array}{l}\text { Mean } \\
\text { difference }\end{array}$ & $\begin{array}{l}\text { Standard error } \\
\text { difference }\end{array}$ & $\begin{array}{c}\text { 95\% confidence } \\
\text { interval }\end{array}$ \\
\hline 11 & 37 & $0.307 \pm 0.605$ & 0.345 & 0.07573 & 0.07812 & 0.08234 & $\mathrm{U}$ & $\mathrm{L}$ \\
21 & 34 & $0.229 \pm 0.258$ & & 0.03233 & & & -0.08 & -0.24 \\
12 & 32 & $0.159 \pm 0.194$ & 0.005 & 0.02437 & -0.11719 & 0.0412 & $\mathrm{U}$ & $\mathrm{L}$ \\
22 & 39 & $0.276 \pm 0.265$ & & 0.03324 & & & -0.19 & -0.03 \\
13 & 33 & $0.173 \pm 0.234$ & 0.625 & 0.02931 & -0.02187 & 0.04464 & $\mathrm{U}$ & $\mathrm{L}$ \\
23 & 25 & $0.195 \pm 0.269$ & & 0.03366 & & & -0.11 & 0.06 \\
\hline
\end{tabular}

SD, standard deviation. $p$ value $\leq 0.05$

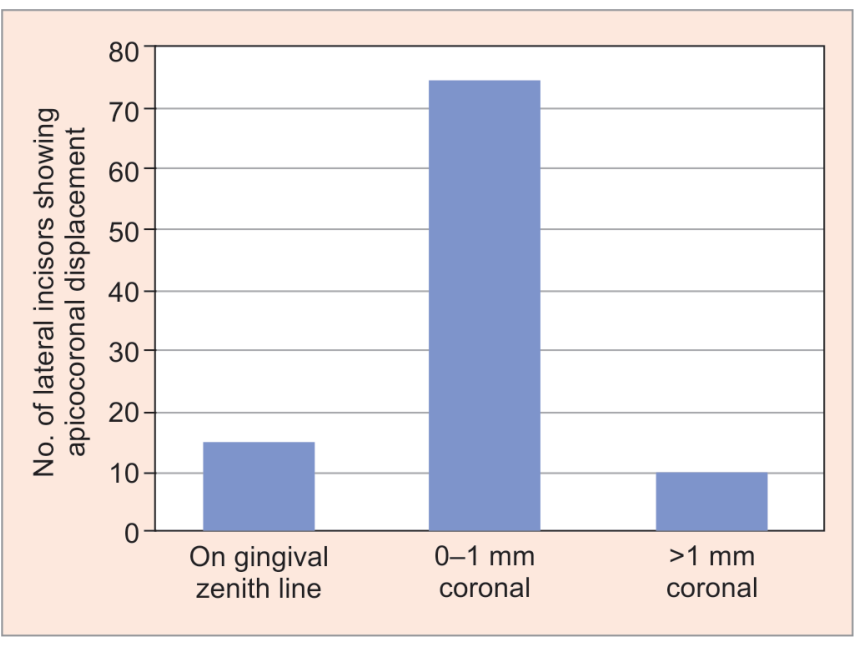

Fig. 5: Apicocoronal displacement of gingival zenith point of lateral incisor from gingival zenith level

Figure 5 shows apicocoronal displacement of GZP of the lateral incisor from the GZL. In the majority of cases (75\%), GZP was displaced in the range of 0 to $1 \mathrm{~mm}$ and in $10 \%$ of cases GZP was displaced $>1 \mathrm{~mm}$ from the GZL and in around $15 \%$ of cases, GZP coincided with the GZL.

Table 2 shows the mean apicocoronal displacement of GZP of lateral incisor with respect to the GZL. The right lateral incisor
Table 2: Mean apicocoronal displacement of lateral incisors from the gingival zenith level

\begin{tabular}{llc}
\hline $\begin{array}{l}\text { Tooth } \\
\text { number }\end{array}$ & $\begin{array}{l}\text { Total number } \\
\text { of teeth }\end{array}$ & $\begin{array}{l}\text { Mean apicocoronal displacement of } \\
\text { lateral incisor from gingival zenith level }\end{array}$ \\
\hline 12 & 50 & $0.606 \pm 0.417$ \\
22 & 50 & $0.537 \pm 0.414$ \\
\hline
\end{tabular}

12-right lateral incisor, 22-left lateral incisor

showed a deviation of $0.606 \pm 0.417 \mathrm{~mm}$ and the left lateral incisor showed a deviation of $0.537 \pm 0.414 \mathrm{~mm}$.

Figure 6 shows no. of teeth showing deviation of GZ from VBM in each of the maxillary anteriors. Out of 50 each, the left lateral incisor had a maximum no of teeth showing deviation from the VBM, while the left canine had the minimum no of teeth showing deviation from the VBM.

\section{Discussion}

Healthy gingiva is among the prime requirement during esthetic treatment planning and it is important to consider its morphology and contour. ${ }^{5}$ To provide more satisfactory esthetic results in a complete rehabilitation involving interdisciplinary intervention; one needs to be very specific about the dentogingival interface. Gingival zenith being an important feature in gingival morphology; can lead to a negative smile effect if there is any discrepancy in GZ position. ${ }^{6}$

Locating GZ is important as it determines the axial inclination of the tooth and therefore plays an important role while trying to 


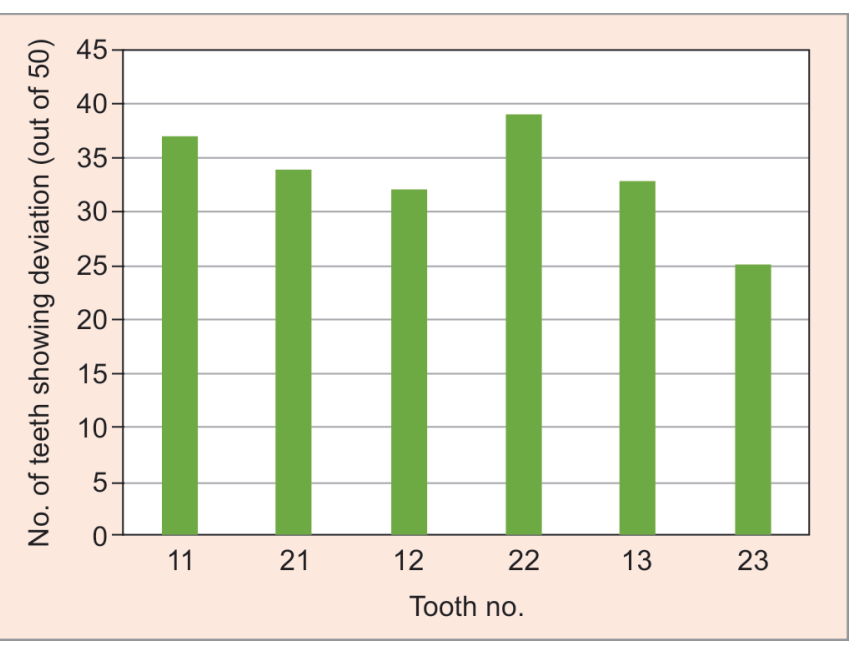

Fig. 6: No. of teeth showing deviation of gingival zenith from the vertical bisected midline (out of 50)

change the mesial-distal dimension of the tooth, diastema closure, and other esthetic procedures. In the present study, GZP and VBM were located using the method described by Chu and Stappert. ${ }^{4}$

In this study, maximum GZ deviation from the VBM was shown by central incisors and least by canines and this was similar to the results obtained in similar studies conducted by Mattos et al. ${ }^{7}$ and by Rathod et al. ${ }^{2}$

In the present study, both mesial and distal deviations of GZP from the VBM were seen in all the teeth but the majority of teeth showed distal deviation. These results were comparable with the results obtained by Appukutan et al. ${ }^{8}$ where a study was conducted to assess the GZ deviation from the VBM. In the same study, the authors reported that mesial deviation was seen i.r.t central and lateral incisors and distal deviation i.r.t canine. It was also comparable to the study done by Goodlin ${ }^{9}$ where it was reported that in the case of central incisor the GZ lies at the distal third, for lateral incisor at the VBM and for canine, it varies between the anterior third to the distal third of the crown.

This was contradictory to the results obtained by Magne and Belser where they reported a distal deviation of GZ of all the maxillary anterior teeth. ${ }^{5}$ But Mattos et al. ${ }^{7}$ and Patil et al. ${ }^{10}$ reported that the GZ is not universally displaced towards the distal aspect. Mattos et al. ${ }^{7}$ also reported that the frequency and magnitude of distal displacement is tooth dependent and is maximum in central incisors and least in canine which was consistent with the results of the present study.

Regarding the apicocoronal displacement of right and left lateral incisors from the GZL, the right lateral incisor showed slightly more displacement than the left lateral incisor, although the difference was not statistically significant. In $15 \%$ of cases, the GZP coincided with the GZL while $75 \%$ of cases showed displacement in the range of 0 to $1 \mathrm{~mm}$, and $10 \%$ of cases showed displacement $>1 \mathrm{~mm}$. Pawar et al. ${ }^{11}$ conducted a study on the GZ of maxillary anterior teeth on 35 mounted maxillary casts and reported that the $\mathrm{GZ}$ of $17 \%$ of lateral incisors was on or below the gingival line which is consistent with the results of the present study. ${ }^{11}$

The proper positioning of the $\mathrm{GZ}$ is essential for balancing the white and pink esthetics and also influences the emergence profile and axial inclination of teeth. ${ }^{10}$ In a study conducted by Kolte et al. in 2020 comparing the lip length and gingival smile line between males and females, it was observed that there was a statistically significant difference in the lip length between males and females with males having a higher lip length than females during smiling. It was also noted that $26.6 \%$ of females and $11.6 \%$ of males exhibited a high gingival smile line. These data suggest that a prominent amount of the population, especially females display a significant portion of their gingiva during smiling and thus it highlights the importance of this study which describes the GZ position and its deviations in the anterior dentition and its importance in smile designing. $^{12}$

Another esthetic soft tissue parameter that is influenced by the $G Z$ is the gingival line angle, formed by the intersection of GZL with the maxillary midline. In a study comparing gingival line angle with gingival smile line, a significant difference was seen between right and left side for male patients while an insignificant difference was seen for female patients and this gender difference should be considered while reconstructing the soft tissues. ${ }^{13}$

The homogeneity in the esthetic perception of smile between layman and expert was highlighted by a study conducted by Pham et al. in 2021. The authors also stressed the need to substitute symmetry with balance and harmony in esthetic dentistry. ${ }^{14}$ Thus, it is important to have in-depth knowledge regarding the gingival parameters for a successful soft tissue rehabilitation.

\section{Conclusion}

Within the limitations of the study, it is observed that the GZ deviation can be mesial or distal and is tooth dependent. The maximum deviation is seen in the central incisor followed by the lateral incisor and canine. The deviation of the GZP from the VBM in comparison to contralateral teeth is comparable except in the case of lateral incisor where the difference was found to be statistically significant. It was found that in the majority of cases, lateral incisors showed coronal displacement of GZ from the GZL.

\section{References}

1. Frizzera F, Tonetto M, Cabral G, et al. Periodontics, implantology, and prosthodontics integrated: the zenith-driven rehabilitation. Case Repor Dentis 2017;2017:1070292. DOI: 10.1155/2017/1070292.

2. Rathod Varsha J. Quantitative evaluation of deviation of gingival zenith position in maxillary anterior dentition. Int J Recent Sci Res 2017;8(4):16741-16744.

3. Bhuvaneswaran M. Principles of smile design. J Conservat Dentis 2010;13(4):225-232. DOI: 10.4103/0972-0707.73387.

4. Chu SJ, Tan JH-P, Stappert CFJ, et al. Gingival zenith positions and levels of the maxillary anterior dentition. J Esthet Restor Dent 2009;21(2):113-121. DOI: 10.1111/j.1708-8240.2009.00242.x.

5. Magne $\mathrm{P}$, Belser $\mathrm{U}$. Bonded porcelain restorations in the anterior dentition: a biomimetic approach. Chicago: Quintessence Publishing; 2002. pp. 57-98.

6. Singhal M, Singhal RA. CAD-CAM prosthodontic option and gingival zenith position for a rotated maxillary right central incisor: an evaluation. Indian J Dent Res 2012;23(1):112-115. DOI: 10.4103/09709290.99053.

7. Mattos CML, Santana RB. A quantitative evaluation of the spatial displacement of the gingival zenith in the maxillary anterior dentition. J Periodontol 2008;79(10):1880-1885. DOI: 10.1902/ jop.2008.080053. 
8. Appukutan D, Cholan PK, Ashwini CR, et al. Assessment of gingival zenith position and distance from vertical bisecting midline in the maxillary anterior dentition-an observational study. J Orofac Sci 2018;10(79):1880-1885.

9. Goodlin R. Gingival aesthetics: a critical factor in smile design. Oral Health and Dental Practice 2003;93(4):1027 Charruel S, Perez C, Foti B. Parameters for gingival contour assessment. J Periodontol 2008;79(5):795-1801.

10. Patil AS, Ranganath V, Yerawadekar SA, et al. Pink esthetics: a study on significant gingival parameters. J Contemp Dent Pract 2020;21(2):207-210. DOI: 10.5005/jp-journals-10024-2739.
11. Pawar B, Mishra $P$, Banga $P$, et al. Gingival zenith and its role in redefining esthetics: a clinical study. J Indian Soc Periodontol 2011;15(2):135-138. DOI: 10.4103/0972-124X.84382.

12. Kolte AP, Kolte RA, Samarth GK. Association of the nasolabial angle and lip length with the gingival and interdental smile line-a gender based evaluation. J Esthet Restor Dent 2020. 1-7.

13. Kolte RA, Kolte AP, Rathi P. Association of the gingival line angle with the gingival and interdental smile line: a gender based evaluation. $J$ Esthet Restor Dent 2019;1-7.

14. Pham TA, Nguyen PA. Morphological features of smile attractiveness and related factors influence perception and gingival aesthetic parameters. Int Dent J 2021;1-9. DOI: 10.1016/j.identj.2021.02.001. 